\title{
Lernweltforschung
}

\section{Band 26}

\section{Herausgegeben von}

H. von Felden, Mainz, Deutschland

R. Egger, Graz, Österreich

Weitere Bände in dieser Reihe

http://www.springer.com/series/12485 
Ausrichtung und Zielsetzung

Innerhalb der derzeit dominierenden gesellschaftlichen Entwicklungen wird der Stellenwert der individuellen Handlungsfähigkeit der sozialen Akteure in den Vordergrund gerückt. Schlagworte wie „Wissensgesellschaft“ oder „Civil Society“ weisen auf die zentrale Bedeutung von Lern- und Bildungsprozessen für die politische, ökonomische und kulturelle Entwicklung hin. Diese Entwicklung schlägt entsprechend auf die einzelnen Biografien durch.

Mit dem in dieser Reihe entfalteten Programm der Lernweltforschung werden diesbezüglich die hier eingelagerten Vielschichtigkeiten und Eigenwilligkeiten, die überraschenden Umgestaltungen und Suchbewegungen von Subjekten in Lernund Bildungsprojekten untersucht. Die hier sichtbar werdenden eigensinnigen Aneignungsprozesse werden innerhalb der je konkreten Situationen und Strukturen analysiert. Lernwelten werden dabei zumindest in einer doppelten Bedeutung sichtbar: Sie sind Rahmen und Rahmungen zugleich, Blick und Gegenblick, in denen Erfahrungen (im Rückgriff auf ein System von Regeln) bewertet, als Bestandteile der sozialen Welt durch subjektive Bedeutungszuweisung (re-)konstruiert werden, und in denen auch das „Aneignungssystem“ selbst und der Prozess der Erfahrungsaufschichtung zur Disposition stehen.

\section{Herausgegeben von}

Heide von Felden

Rudolf Egger

Johannes-Gutenberg-Universität

Karl-Franzens-Universität

Mainz, Deutschland

Graz, Österreich 
Steirische Hochschulkonferenz

(Hrsg.)

Qualität in

Studium und Lehre

Kompetenz- und Wissensmanagement im steirischen Hochschulraum

䭪 Springer VS 
Steirische Hochschulkonferenz

Verantwortlicher Herausgeber: Rudolf Egger

Karl-Franzens-Universität Graz

Österreich

Lernweltforschung

ISBN 978-3-658-13737-3 ISBN 978-3-658-13738-0 (eBook)

DOI 10.1007/978-3-658-13738-0

Die Deutsche Nationalbibliothek verzeichnet diese Publikation in der Deutschen Nationalbibliografie; detaillierte bibliografische Daten sind im Internet über http://dnb.d-nb.de abrufbar.

\section{Springer VS}

(C) Springer Fachmedien Wiesbaden 2016

Das Werk einschließlich aller seiner Teile ist urheberrechtlich geschützt. Jede Verwertung, die nicht ausdrücklich vom Urheberrechtsgesetz zugelassen ist, bedarf der vorherigen Zustimmung des Verlags. Das gilt insbesondere für Vervielfältigungen, Bearbeitungen, Übersetzungen, Mikroverfilmungen und die Einspeicherung und Verarbeitung in elektronischen Systemen.

Die Wiedergabe von Gebrauchsnamen, Handelsnamen, Warenbezeichnungen usw. in diesem Werk berechtigt auch ohne besondere Kennzeichnung nicht zu der Annahme, dass solche Namen im Sinne der Warenzeichen- und Markenschutz-Gesetzgebung als frei zu betrachten wären und daher von jedermann benutzt werden dürften.

Der Verlag, die Autoren und die Herausgeber gehen davon aus, dass die Angaben und Informationen in diesem Werk zum Zeitpunkt der Veröffentlichung vollständig und korrekt sind. Weder der Verlag noch die Autoren oder die Herausgeber übernehmen, ausdrücklich oder implizit, Gewähr für den Inhalt des Werkes, etwaige Fehler oder Äußerungen.

Lektorat: Stefanie Laux, Stefanie Loyal

Gedruckt auf säurefreiem und chlorfrei gebleichtem Papier

Springer VS ist Teil von Springer Nature

Die eingetragene Gesellschaft ist Springer Fachmedien Wiesbaden GmbH 


\section{Inhalt}

\section{Kontexte}

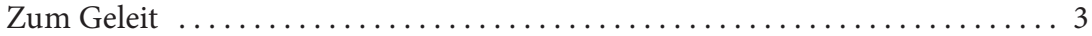

Elisabeth Freismuth

Hochschuldidaktische Herausforderungen zwischen Bologna und

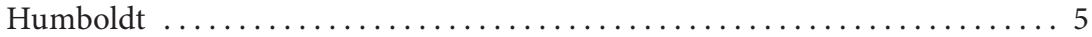

Elgrid Messner

Hochschuldidaktik im Kontext lebenslangen Lernens . . . . . . . . . . . 9

Rudolf Egger

Hochschuldidaktik und Organisationsentwicklung. Über den

Zusammenhang institutioneller Rahmenbedingungen zur Steigerung

der Lehrqualität

Daniela Wagner

Forschende Lehrende als akademisches Selbstverständnis.

Professionalisierung durch Forschung an den Pädagogischen

Hochschulen

Georg Tafner

Didaktik-Ethik. Wie können Hochschullehrende ihre Studierenden bestmöglich fördern, damit diese befähigt werden ihr „Akademisches Gelöbnis“ nach Studienende erfüllen zu können? Ein Versuch . 
Ein Lehrkompetenzmodell für den Steirischen Hochschulraum.

Voraussetzungen, Rahmenbedingungen und Perspektiven ........... 121

Gudrun Salmhofer

Hochschuldidaktik - Herausforderungen in der Weiterentwicklung.

Überlegungen mit besonderem Fokus auf die Universitäten nach

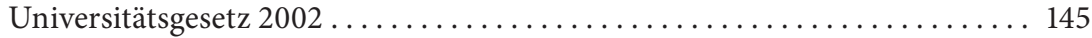

Andrea Geisler

\section{Orte}

Das DiZ - Zentrum für Hochschuldidaktik. Eine Erfolgsgeschichte

Franz Waldherr

„Wer ewig forschend sich bemüht“. Ausbildung und Wissenschaft:

Theorie und Praxis an der KPH Graz ..................... 187

Katharina Ogris

Hochschuldidaktik an der Pädagogischen Hochschule Steiermark:

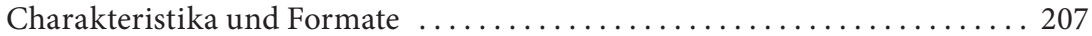

Marlies Matischek-Jauk, Martin Teufel und Elisabeth Amtmann

Instrumental- und Gesangspädagogik zwischen Theorie und Praxis.

Reflexionen zum ersten Didaktik-Tag an der Kunstuniversität Graz .... 225

Magdalena Krinner, Silke Kruse-Weber und Cristina Marin

\section{Formate}

Die Qualität der Hochschullehre und deren Messung $\ldots \ldots \ldots \ldots \ldots \ldots 243$ Doris Kiendl-Wendner

Möglichkeiten und Grenzen der Evaluation der Lehre ............. 263 Nina Bialowas

Auseinandersetzung mit der Qualität didaktischer E-Learning Szenarien . 283 Jutta Pauschenwein, Anastasia Sfiri und Irmgard Schinnerl-Beikircher 
Entwicklung von „eDidactics“: Ein Fortbildungsprogramm für den Einsatz von Technologien in der Hochschullehre ... 305 Michael Kopp, Martin Ebner, Herwig Rehatschek, Patrick Schweighofer, Anastasia Sfiri, Birgit Swoboda und Martin Teufel

Rolle und Herausforderungen von Online-Kursen (MOOCs) für die Hochschullehre ..................................... 323 Martin Ebner, Michael Kopp und Alexandra Dorfer-Novak 